\title{
Cinétique de dégradation in sacco de tiges de maïs normal et bm3
}

\author{
MR Tovar-Gómez 1, JC Emile 1, B Michalet-Doreau 2, Y Barrière 1 \\ 'INRA, Station d'Amélioration des Plantes Fourragères, 86600 Lusignan; 2INRA, Valeur Alimentaire, \\ Theix, 63122 St-Genes-Champanelle, France
}

\begin{abstract}
La valeur alimentaire du maïs fourrage peut être améliorée en prenant en compte le potentiel des génotypes brown-midrib $(\mathrm{bm})$. L'objectif de cette étude a donc été la comparaison des cinétiques de dégradation des constituants pariétaux de tiges de maïs normal $(\mathrm{N})$ et de son isogènique brown-midrib (bm3).
\end{abstract}

Les parties de tiges de maïs situées entre la base et le noeud portant l'épi ont été récoltées au stade ensilage ( $30-35 \%$ MS, plante entière). Ces tiges ont été séchées et broyées $(2 \mathrm{~mm})$. Les mesures de dégradation in sacco ont été réalisées sur 4 vaches laitières taries de race Holstein $(713 \mathrm{~kg})$ munies d'une fistule du rumen. Les animaux ont reçu un régime distribué en 2 repas égaux composé de $60 \%$ de foin de graminée et de $40 \%$ de concentré $(1,3 \% \mathrm{PV})$. Nous avons utilisé des sachets en tissu de nylon (ANKOM, $20 \mathrm{~cm} \times 10 \mathrm{~cm}, 52$ $\pm 2 \mu \mathrm{m}, 15 \mathrm{mg} / \mathrm{cm} 2$ ). Pour chaque hybride, nous avons réalisé 10 points de cinétique $(2,4$, $6,8,12,18,24,48,72$ et 96 h) et 2 répétitions par animal.

Sur l'aliment initial et sur les résidus après incubation, nous avons déterminé les teneurs en constituants pariétaux selon la méthode de Goering et Van Soest (1970, Agric Handbook No. 379, Agric Res Serv, USA, 19). Les

Paramètre

Matière sèche (MS)

$\begin{array}{lcccc} & \mathrm{N} & \text { bm3 } & \text { mse } & \\ \text { A (\%) } & 24,5 & 9,3 & 2,6 & \text { ** } \\ \text { a (\%) } & 44,5 & 60,3 & 0,7 & \text { *** } \\ \text { b (\%) } & 31,0 & 30,4 & 3,1 & \text { ns } \\ \text { c (\%/h) } & 2,9 & 3,9 & 0,9 & \text { ns } \\ \text { t (h) } & 8,0 & 7,8 & 1,5 & \text { ns } \\ \text { DT }(\%) & 50,7 & 67,8 & 0,5 & \text { *** }\end{array}$

cinétiques de dégradation de la MS et des constituants de la MS ont été ajustées à un modèle de type exponentiel dépendant du temps t (Dhanoa, 1988, Grass For Sci, 43, $441-444$ ), et la dégradabilité théorique (DT) a été calculée avec un taux de passage des particules dans le rumen de $0,06 / \mathrm{h}$.

Les teneurs en parois totales (NDF), en lignocellulose $(A D F)$ et en lignine ( $A D L$ ) sont respectivement de 170,116 et $31 \mathrm{~g} / \mathrm{kg}$ MS plus faibles chez l'hybride bm3 comparé au normal. La DT plus élevée de la MS (+ 17,1 points) et de la fraction NDF (+ 7,2 points) de l'hybride bm3 par rapport au mais normal est essentiellement liée à une diminution de la fraction indégradable (A), au bénéfice de la fraction rapidement (a) ou lentement dégradable (b) respectivement pour la MS et la fraction pariétale. Les vitesses de dégradation (c) ne sont pas modifiées. Des résultats similaires à ceux obtenus pour la fraction NDF ont été trouvés avec la fraction ADF.

En conclusion, l'augmentation de la DT de la MS du mais bm3 par rapport au maïs normal serait liée à la diminution de la teneur en parois, mais aussi de la lignification de ces parois comme le suggère l'augmentation de la DT du NDF de cet hybride.

ns : non significatif, ${ }^{\star}: P<0,05,{ }^{\star \star}: P<0,01,{ }^{* \star \star} P<0,001$

\begin{tabular}{cccc}
\multicolumn{4}{c}{ Neutral Detergent Fiber (NDF) } \\
$\mathrm{N}$ & bm3 & mse & \\
& & & \\
30,3 & 15,9 & 4,5 & * \\
19,6 & 20,8 & 0,9 & ns \\
50,1 & 63,4 & 5,1 & * \\
2,7 & 3,7 & 0,6 & ns \\
8,6 & 7,7 & 1,4 & ns \\
28,9 & 36,1 & 0,4 & $* \star *$
\end{tabular}

